PROCEEDINGS OF THE

AMERICAN MATHEMATICAL SOCIETY

Volume 132, Number 12, Pages 3731-3739

S 0002-9939(04)07493-3

Article electronically published on July 12, 2004

\title{
ON THE GAUSS MAP OF HYPERSURFACES WITH CONSTANT SCALAR CURVATURE IN SPHERES
}

\author{
HILÁRIO ALENCAR, HAROLD ROSENBERG, AND WALCY SANTOS
}

(Communicated by Richard A. Wentworth)

\begin{abstract}
In this work we consider connected, complete and orientable hypersurfaces of the sphere $\mathbb{S}^{n+1}$ with constant nonnegative $r$-mean curvature. We prove that under subsidiary conditions, if the Gauss image of $M$ is contained in a closed hemisphere, then $M$ is totally umbilic.
\end{abstract}

\section{INTRODUCTION}

One of the most celebrated theorems of minimal surfaces in $\mathbb{R}^{3}$ is Bernstein's theorem:

Theorem (Bernstein [4]). Let $M \subset \mathbb{R}^{3}$ be a complete minimal surface in $\mathbb{R}^{3}$ that is given by an entire (defined over the whole $\mathbb{R}^{2}$ ) graph of a smooth function $f$ : $\mathbb{R}^{2} \rightarrow \mathbb{R}$. Then $M$ is a plane.

The above result is also true under the weaker hypothesis that the image of the Gauss map of $M$ lies in an open hemisphere of $\mathbb{S}^{n+1}$, as one can see in [3]. These results raise the following problem for the geometry of minimal surfaces in spheres: Does there exist a similar result for minimal hypersurfaces of the unit sphere? The answer to this question was obtained independently by E. De Giorgi ([6]) and J. Simons (see [13] - Theorem 5.2.1) as follows.

Theorem. If the Gauss image (see the definition below) of a compact minimal hypersurface $M^{n}$ in the Euclidean sphere lies in an open hemisphere of $\mathbb{S}^{n+1}$, then $M$ must be a great hypersphere in $\mathbb{S}^{n+1}$.

After that, K. Nomizu and Brian Smyth (see 9] - Theorem 2) were able to generalize this result to constant mean curvature hypersurfaces of $\mathbb{S}^{n+1}$, proving the following result:

Theorem (Nomizu-Smyth). Let $M$ be any compact connected orientable manifold of dimension $n \geq 2$ immersed in the sphere $\mathbb{S}^{n+1}$ with constant mean curvature. If the Gauss image of $M$ lies in a closed hemisphere of $\mathbb{S}^{n+1}$, then $M$ is a hypersphere in $\mathbb{S}^{n+1}$.

Received by the editors April 28, 2003 and, in revised form, September 1, 2003.

2000 Mathematics Subject Classification. Primary 53C40; Secondary 53A10.

Key words and phrases. r-mean curvature, spheres, Gauss image.

The first and third authors' research was partially supported by CNPq and the French-Brazilian Agreement in Mathematics. 
The goal of this work is to extend these results to higher-order constant mean curvature hypersurfaces of the sphere. First let us fix some notation.

Let $M^{n}$ be a compact orientable Riemannian manifold and let $x: M^{n} \rightarrow \mathbb{S}^{n+1}$ be an isometric immersion into the unit sphere $\mathbb{S}^{n+1} \subset \mathbb{R}^{n+2}$. Since $M$ is orientable, we can choose a global unit normal field $N$. The Riemannian connections $\nabla$ and $\widetilde{\nabla}$ of $M$ and $\mathbb{S}^{n+1}$, respectively, are related by

$$
\widetilde{\nabla}_{X} Y=\nabla_{X} Y+\langle A(X), Y\rangle N
$$

where $A$ is the shape operator of the immersion, defined by

$$
\widetilde{\nabla}_{X} N=-A(X) \text {. }
$$

Let $k_{1}, \ldots, k_{n}$ be the eigenvalues of $A$. We define the $r$-mean curvature of the immersion at a point $p$ by

$$
H_{r}=\frac{1}{\left(\begin{array}{l}
n \\
r
\end{array}\right)} \sum_{i_{1}<\ldots<i_{r}} k_{i_{1}} \ldots k_{i_{r}}=\frac{1}{\left(\begin{array}{l}
n \\
r
\end{array}\right)} S_{r},
$$

where $S_{r}$ is the $r$-symmetric function of the $k_{1}, \ldots, k_{n}$. In order to unify the notation, we will define $H_{0}=1$ and $H_{r}=0$, for all $r \geq n+1$. For $r=1, H_{1}=H$ is the mean curvature of the immersion, in the case $r=2, H_{2}$ is the scalar curvature and for $r=n, H_{n}$ is the Gauss-Kronecker curvature.

The Gauss map $\phi: M^{n} \rightarrow \mathbb{S}^{n+1}$ is defined by

$$
\phi(P)=N(P) \in \mathbb{S}^{n+1} .
$$

The set $\phi(M)$ is called the Gauss image of $M$. We observe that the Gauss image depends on the choice of the orientation of $M$, but the two possibilities are related by an antipodal mapping of $\mathbb{S}^{n+1}$. Thus the statement that the Gauss image of $M$ is contained in a closed hemisphere of $\mathbb{S}^{n+1}$ is independent of the orientation of $M$.

For the case $H_{r}=0$, we obtain that

Theorem A. Let $M^{n} \rightarrow \mathbb{S}^{n+1}$ be a compact and connected hypersurface of $\mathbb{S}^{n+1}$ with $H_{r}=0$, for some $r=1, \ldots, n-1$. Assume that the Gauss image of $M$ is contained in a closed hemisphere and that $H_{r-1}$ does not change sign in $M$. Then $M$ is totally geodesic.

If $H_{r}>0$, we were able to prove that

Theorem B. Let $M^{n} \rightarrow \mathbb{S}^{n+1}$ be a compact and connected hypersurface of $\mathbb{S}^{n+1}$ with constant positive $(r+1)$-mean curvature $H_{r+1}$, for some $r=0, \ldots, n-2$. Assume that the Gauss image of $M$ is contained in a closed hemisphere, $H_{r} \geq 0$ and that the following inequality holds:

$$
H_{1} H_{r} \geq H_{r+1} \text {. }
$$

Then $M$ is totally umbilic.

In the case of the scalar curvature, part of the hypothesis of the above theorems is trivially satisfied, and we obtain the following result.

Theorem C. Let $M^{n}$ be a compact orientable hypersurface of the sphere with constant scalar curvature $H_{2} \geq 0$. In the case $H_{2}=0$, suppose also that $H_{1}$ does not change sign. If the Gauss image of $M$ lies in a closed hemisphere of $\mathbb{S}^{n+1}$, then $M$ is totally umbilic. 
The authors do not know if the hypotheses of Theorems A, B and $\mathrm{C}$ can be weakened.

Parts of these results were obtained by R. Reilly, [11], with the strong hypothesis that the Gauss image is contained in an open hemisphere.

The first and the third authors would like to thank the hospitality of Institut de Mathématiques de Jussieu, where this work was done.

\section{Preliminaries}

We introduce the $r^{t h}$ Newton tensors, $P_{r}: T_{p} M \rightarrow T_{p} M$, which are defined inductively by

$$
\begin{aligned}
& P_{0}=I, \\
& P_{r}=S_{r} I-A P_{r-1}, r>1 .
\end{aligned}
$$

It is easy to see that each $P_{r}$ commutes with $A$, and if $e_{i}$ is an eigenvector of $A$ associated to the principal curvature $k_{i}$, then

$$
P_{1}\left(e_{i}\right)=\mu_{i} e_{i}=\left(S_{1}-k_{i}\right) e_{i} .
$$

In [11, Reilly showed that the $P_{r}$ 's satisfy the following.

Proposition 1.1 (11], see also 2 - Lemma 2.1). Let $x: M^{n} \rightarrow N^{n+1}$ be an isometric immersion between two Riemannian manifolds, and let $A$ be its second fundamental form. The $r^{\text {th }}$ Newton tensor $P_{r}$ associated to A satisfies:

(1) $\operatorname{trace}\left(P_{r}\right)=(n-r) S_{r}$,

(2) $\operatorname{trace}\left(A P_{r}\right)=(r+1) S_{r+1}$,

(3) $\operatorname{trace}\left(A^{2} P_{r}\right)=S_{1} S_{r+1}-(r+2) S_{r+2}$.

Associated to each Newton tensor $P_{r}$, we define a second-order differential operator

$$
L_{r}(f)=\operatorname{trace}\left(P_{r} \operatorname{Hess} f\right) .
$$

We observe that for $r=0, L_{0}$ is the Laplacian, which is always an elliptic operator. If $N^{n+1}$ has constant sectional curvature, it follows from the Codazzi equation (see 12, p. 225) that $L_{r}$ is

$$
L_{r}(f)=\operatorname{div}_{M}\left(P_{r} \nabla f\right) .
$$

Hence $L_{r}$ is a self-adjoint operator. In general, for $r \geq 1, L_{r}$ is not an elliptic operator. The following proposition give us a condition for $L_{r}$ to be elliptic.

Proposition 1.2. Let $M^{n}$ be a connected, compact and orientable Riemannian manifold, and let $x: M^{n} \rightarrow \mathbb{S}^{n+1}$ be an isometric immersion with $H_{r+1}$ constant. If $M^{n}$ has one point where all principal curvatures are positive, then $L_{r}$ is an elliptic operator.

Proof. See the proof of Proposition 3.2 of [2].

For hypersurfaces of $\mathbb{R}^{n+1}$ with $H_{r}=0$, Hounie and Leite, [8], were able to give a geometric condition that is equivalent to $L_{r}$ being elliptic. In fact, their proof can be generalized to hypersurfaces of the sphere, and we have the following result.

Proposition 1.3 ([8] - Proposition 1.5). Let $M$ be a hypersurface in $\mathbb{R}^{n+1}$ or $S^{n+1}$ with $H_{r}=0,2 \leq r<n$. Then the operator $L_{r-1}(f)=\operatorname{div}\left(P_{r-1} \nabla f\right)$ is elliptic at $p \in M$ if and only if $H_{r+1}(p) \neq 0$. 
Since the $r$-mean curvatures of $M^{n}$ are symmetric means of the $n$-uple of principal curvatures of $M$, they are related by the following algebraic inequalities (see [7], p. 52, and [5], p. 285):

$$
H_{i-1} H_{i+1} \leq H_{i}^{2}, \quad \forall i, 1 \leq i<n .
$$

Also, provided that the $H_{r}$ 's are nonnegative, $r=1, \ldots, i$,

$$
H_{1} \geq H_{2}^{1 / 2} \geq H_{3}^{1 / 3} \geq \ldots \geq H_{i}^{1 / i} .
$$

Furthermore, the equality in (1.1) and (1.2) holds only if $k_{1}=k_{2}=\ldots=k_{n}$.

\section{INTEGRAL FORMULA}

Consider the functions $f, g: M \rightarrow \mathbb{R}$, given by

$$
f(P)=\langle N(P), \alpha\rangle
$$

and

$$
g(P)=\langle x(P), \alpha\rangle,
$$

where $\alpha$ is a fixed vector of $\mathbb{R}^{n+2}$. These functions satisfy (see [2], Lemma 5.2)

$$
\begin{gathered}
L_{r}(g)=-(r+1) S_{r+1} f-(n-r) S_{r} g, \\
L_{r}(f)=-\left(S_{1} S_{r+1}-(r+2) S_{r+2}\right) f-(r+1) S_{r+1} g,
\end{gathered}
$$

where, in the last equation, we use the fact that $S_{r+1}$ is constant. In particular, for $r=0$, we get

$$
\begin{gathered}
\triangle(g)=-S_{1} f-n g, \\
\triangle(f)=-\left(S_{1}^{2}-2 S_{2}\right) f-S_{1} g=-\|A\|^{2} f-S_{1} g .
\end{gathered}
$$

The following integral formula will be needed.

Proposition 2.1. Let $M^{n} \rightarrow \mathbb{S}^{n+1}$ be a compact orientable hypersurface isometrically immersed in $\mathbb{S}^{n+1}$, with $H_{r+1}$ constant, for some $r$ with $0 \leq r<n-2$. Then,

$$
\int_{M}\left[(n-r-1) S_{1} S_{r+1}-n(r+2) S_{r+2}\right] f d M=0 .
$$

Proof. Observe that, since $S_{r+1}$ is constant, by (2.2) and (2.3), we obtain that

$$
\begin{gathered}
L_{r} f-\frac{(r+1)}{n} S_{r+1} \triangle g=-\left(S_{1} S_{r+1}-\left(r+2 S_{r+2}\right) f\right. \\
-(r+1) S_{r+1} g+\frac{(r+1)}{n} S_{r+1} S_{1} f+\frac{(r+1)}{n} S_{r+1} n g \\
=-S_{1} S_{r+1} f+(r+2) S_{r+2} f+\frac{(r+1)}{n} S_{r+1} S_{1} f \\
=\frac{1}{n}\left[-n S_{1} S_{r+1} f+n(r+2) S_{r+2} f+(r+1) S_{r+1} S_{1} f\right] \\
\quad=\frac{1}{n}\left[(-n+r+1) S_{1} S_{r+1} f+n(r+2) S_{r+2} f\right] \\
=\frac{-1}{n}\left[(n-r-1) S_{1} S_{r+1}-n(r+2) S_{r+2}\right] f .
\end{gathered}
$$


Integrating this last expression and applying Stokes' Theorem, one has that

$$
\begin{gathered}
\int_{M}\left[(n-r-1) S_{1} S_{r+1}-n(r+2) S_{r+2}\right] f d M \\
=\int_{\partial M}\left\langle P_{r} \nabla f-\frac{(r+1)}{n} S_{r+1} \nabla g, \nu\right\rangle d S=0,
\end{gathered}
$$

where the last equality follows from the fact that $\partial M=\emptyset$.

\section{THE CASE $H_{r}=0$}

In this section we consider hypersurfaces of the sphere with $H_{r}=0$. We have the following result.

Theorem 3.1 (Theorem A of the Introduction). Let $M^{n} \rightarrow \mathbb{S}^{n+1}$ be a compact and connected hypersurface of $\mathbb{S}^{n+1}$ with $H_{r}=0$, for some $r=1, \ldots, n-1$. Assume that the Gauss image of $M$ is contained in a closed hemisphere and that $H_{r-1}$ does not change sign in $M$. Then $M$ is totally geodesic.

Proof. By (1.1) and the fact that $H_{r}=0$, it follows that

$$
H_{r+1} H_{r-1} \leq 0 .
$$

Thus, since $H_{r-1}$ does not change sign in $M, H_{r+1}$ also does not change sign on $M$.

On the other hand, our hypothesis on the Gauss image implies that there exists a vector $\alpha \in \mathbb{R}^{n+2}$ such that

$$
f(P)=\langle N(P), \alpha\rangle
$$

is nonnegative along $M$. Hence, $f(P) S_{r+1}(P)$ does not change sign along $M$. The equation (2.5), in our case, reads

$$
\int_{M} f(P) S_{r+1}(P) d M=0 .
$$

Thus,

$$
f(P) S_{r+1}(P)=0, \quad \forall P \in M .
$$

Let $\mathcal{A} \subset M$ be the set of all points of $M$ where $S_{r+1}(P)>0$. In $\mathcal{A}$, by equation (3.1), $f \equiv 0$. By continuity, $f$ is zero along $\overline{\mathcal{A}}$, where $\overline{\mathcal{A}}$ is the closure of $\mathcal{A}$. On the other hand, the set $M / \overline{\mathcal{A}}$ is an open set of $M$ where

$$
H_{r}=H_{r+1}=0 \text {. }
$$

Hence equality holds in (1.1), for all $P \in M / \overline{\mathcal{A}}$. This means that all points in $M / \overline{\mathcal{A}}$ are umbilic. That is, for all $P \in M / \overline{\mathcal{A}}$,

$$
k_{1}(P)=\ldots=k_{n}(P)=a(P) .
$$

Thus,

$$
0=S_{r}(P)=a^{r}(P) .
$$

This implies that all points of $M / \overline{\mathcal{A}}$ are totally geodesic, and hence $f$ is constant along each connected component of $M / \overline{\mathcal{A}}$. Since along the boundary of those sets, $f=0$, we conclude that $f$ is identically zero on $M$, that is, $M$ is totally geodesic (see Theorem 1 of 9 ).

Remark. For the case $r=1$, we observe that $S_{r-1}=S_{0}=1$ does not change sign. Hence, the theorem is a generalization of Theorem 2 in [9], in the minimal case. 


\section{THE CASE $H_{r+1}>0$}

Let us consider the case $H_{r+1}>0$. We have the following result:

Theorem 4.1 (Theorem B of the Introduction). Let $M^{n} \rightarrow \mathbb{S}^{n+1}$ be a compact and connected hypersurface of $\mathbb{S}^{n+1}$ with constant positive $(r+1)$-mean curvature $H_{r+1}$, for some $r=0, \ldots, n-2$. Assume that the Gauss image of $M$ is contained in a closed hemisphere, $H_{r} \geq 0$ and that the following inequality holds:

$$
H_{1} H_{r} \geq H_{r+1} \text {. }
$$

Then $M$ is totally umbilic.

Proof. By Proposition [2.1, we have that for a fixed $\alpha \in \mathbb{R}^{n+2}$, the function $f=$ $\langle N(P), \alpha\rangle$ satisfies

$$
\int_{M}\left[(n-r-1) S_{1} S_{r+1}-n(r+2) S_{r+2}\right] f d M=0 .
$$

We are going to prove that the integrand has a fixed sign, for some $\alpha \in \mathbb{R}^{n+2}$. Since the Gauss image of $M$ lies in a closed hemisphere, there exists a vector $\alpha \in \mathbb{R}^{n+2}$ such that

$$
f(P)=\langle N(P), \alpha\rangle \geq 0, \quad \forall P \in M .
$$

On the other hand, the relation $H_{1} H_{r} \geq H_{r+1}$ implies that $H_{1} H_{r+1} \geq H_{r+2}$. In fact, by using equation (1.1), one has that

$$
H_{r} H_{r+2} \leq H_{r+1}^{2} \leq H_{r} H_{1} H_{r+1} .
$$

Observe that $H_{r} \neq 0$; otherwise, the last inequality implies that $H_{r}$ and $H_{r+1}$ are equal to zero, which is a contradiction. Hence, $H_{r}>0$ and we can divide (4.4) by

$$
H_{1} H_{r+1} \geq H_{r+2} \text {. }
$$

Since

by (4.5), one has

$$
H_{i}=\frac{S_{i}}{\left(\begin{array}{c}
n \\
i
\end{array}\right)}
$$

This implies that

$$
\frac{S_{1}}{n} \frac{S_{r+1}}{\left(\begin{array}{c}
n \\
r+1
\end{array}\right)} \geq \frac{S_{r+2}}{\left(\begin{array}{c}
n \\
r+2
\end{array}\right)}
$$

$$
(n-r-1) S_{1} S_{r+1}-n(r+2) S_{r+2} \geq 0 .
$$

The inequalities (4.3) and (4.6) imply that

$$
\left[(n-r-1) S_{1} S_{r+1}-n(r+2) S_{r+2}\right] f \geq 0 .
$$

Thus, by 4.2), we have that

$$
\left[(n-r-1) S_{1} S_{r+1}-n(r+2) S_{r+2}\right] f=0 .
$$

Observe that the function $f$ is not identically zero, since in this case, $M$ has to be totally geodesic (see Theorem 1 of [9]) and hence $H_{r}=0$, which is a contradiction. Let $\mathcal{B} \subset M$ be the open and nonempty set where $f>0$. Along $\mathcal{B}$, we have

$$
(n-r-1) S_{1} S_{r+1}-n(r+2) S_{r+2}=0,
$$

that is, equality holds in (4.6). This means that equality also holds in (1.1), since this inequality was used to obtain (4.6). Hence, all points of $\mathcal{B}$ are umbilic. In this 
case, $M$ has an elliptic point and $S_{r}=$ constant $>0$. Thus, by Proposition 1.2 the operator $L_{r}$ is an elliptic operator. By the principle of analytic continuation, since $M$ is totally umbilic in an open set, it has to be totally umbilic.

Observe that in the case $r=2$, part of the hypotheses of Theorems 3.1 and 4.1 is trivially satisfied, and we have the following result.

Corollary 4.1 (Theorem $\mathrm{C}$ of the Introduction). Let $M^{n}$ be a compact orientable hypersurface of the sphere with constant scalar curvature $H_{2} \geq 0$. In the case $H_{2}=0$, suppose also that $H_{1}$ does not change sign. If the Gauss image of $M$ lies in a closed hemisphere of $\mathbb{S}^{n+1}$, then $M$ is totally umbilic.

Proof. The case $H_{2}=0$ is the statement of Theorem 3.1 For the case $H_{2}>0$, the hypothesis (4.1) in Theorem 4.1 reads

$$
H_{1}^{2} \geq H_{2}
$$

which is always true by equation (1.1). The above equation also says that $H_{1}$ is different from zero on $M$. Hence we can choose the orientation of $M$ so that $H_{1}>0$. The sign of $\mathrm{H}_{2}$ does not depend on the orientation; thus the result follows directly from Theorem 4.1

We now give conditions that imply condition (4.1). First of all, if $H_{i}$ is nonnegative for $i=1, \ldots, r-1$, then (4.1) holds. This fact was stated in [12], p. 232, and we are including its proof here for the sake of completeness. Let $\left(x_{1}, \ldots, x_{n}\right)$ be an $n$-uple of real numbers, and let $S_{r}$ be the $r$-symmetric function of the $x_{1}, \ldots, x_{n}$. Let $H_{r}$ be defined by

$$
H_{r}=\frac{1}{\left(\begin{array}{l}
n \\
r
\end{array}\right)} S_{r}=\frac{1}{\left(\begin{array}{l}
n \\
r
\end{array}\right)} \sum_{i_{1}<\ldots<i_{r}} x_{i_{1}} \ldots x_{i_{r}} .
$$

Proposition 4.1. With the above notation, if $H_{i} \geq 0$ for all $i=1, \ldots, r-1$, then

$$
H_{1} H_{i+1} \geq H_{i+2}, \quad \forall i=1, \ldots, r-1 .
$$

Moreover,

$$
(n-i-1) S_{1} S_{i+1}-n(i+2) S_{i+2} \geq 0, \quad \forall i=1, \ldots, r-1 .
$$

Proof. By using (1.1), we have that

$$
H_{r} H_{r-2} \geq H_{r-1}^{2} \geq 0
$$

and

$$
H_{r+1} H_{r-1} \geq H_{r}^{2} \geq 0 .
$$

Since $H_{r-2}$ and $H_{r-1}$ are nonnegative, it follows that $H_{r} \geq 0$ and $H_{r+1} \geq 0$. Let us prove (4.7). We will argue by induction on $i$. By using (1.1), with $i=1$, and the fact that $H_{0}=1$, we obtain

$$
H_{1}^{2} \geq H_{0} H_{2}=H_{2}
$$

Hence (4.7) holds for $i=0$. By induction, let us suppose that

$$
H_{1} H_{i} \geq H_{i+1} \text {. }
$$

This implies, using equation (1.1), that

$$
H_{i} H_{i+2} \leq H_{i+1}^{2} \leq H_{i+1} H_{1} H_{i} .
$$


If $H_{i}=0$, then (4.9) implies that $H_{i+1} \leq 0$. Since $H_{i+1} \geq 0$, it follows that $H_{i+1}=0$. Thus we have equality in (1.2), which implies that $x_{k}=0, \forall k=1, \ldots, n$. Hence (4.7) holds in this case.

Let us suppose $H_{i}>0$. In this case, we can divide 4.10 by $H_{i}$ and obtain

$$
H_{1} H_{i+1} \geq H_{i+2},
$$

and we finish the proof of (4.7). In order to obtain (4.8), just observe that

$$
H_{i}=\frac{S_{i}}{\left(\begin{array}{c}
n \\
i
\end{array}\right)} .
$$

Then, by (4.11), one has

This implies that

$$
\frac{S_{1}}{n} \frac{S_{i+1}}{\left(\begin{array}{c}
n \\
i+1
\end{array}\right)} \geq \frac{S_{i+2}}{\left(\begin{array}{c}
n \\
i+2
\end{array}\right)}
$$

$$
(n-i-1) S_{1} S_{i+1}-n(i+2) S_{i+2} \geq 0, \quad \forall i=1, \ldots, r-2 .
$$

Thus, we have the following result.

Corollary 4.2. Let $M^{n} \rightarrow \mathbb{S}^{n+1}$ be a compact and connected hypersurface of $\mathbb{S}^{n+1}$ with constant positive $r$-mean curvature $H_{r}$, for some $r=1, \ldots, n-1$. Assume that the Gauss image of $M$ is contained in a closed hemisphere and that $H_{i} \geq 0$ for all $i=1, \ldots, r-1$. Then $M$ is totally umbilic.

In the following proposition (see Proposition 2.3 in [2]) we have another geometric condition that gives $H_{i} \geq 0$ for all $i=1, \ldots, r-1$.

Proposition 4.2. Let $M^{n}$ be a connected compact Riemannian manifold, and let $x: M^{n} \rightarrow \mathbb{S}^{n+1}$ be an isometric immersion. If $H_{r}>0$ and $x(M)$ is contained in an open hemisphere of $\mathbb{S}^{n+1}$, then $H_{i}>0$ for all $i=1, \ldots, r-1$.

This and Corollary 4.2 imply

Corollary 4.3. Let $x: M^{n} \rightarrow \mathbb{S}^{n+1}$ be an isometric immersion of a compact and connected hypersurface of $\mathbb{S}^{n+1}$ with constant positive $r$-mean curvature $H_{r}$, for some $r=1, \ldots, n-1$. Assume that the Gauss image of $M$ is contained in a closed hemisphere and that $x(M)$ is contained in an open hemisphere of $\mathbb{S}^{n+1}$. Then $M$ is totally umbilic.

\section{REFERENCES}

1. Alencar, H., do Carmo, M., and Colares, A.- Stable hypersurfaces with constant scalar curvature, Math. Z. 213, 117-131 (1993). MR 94d:53080

2. Barbosa, J.L. and Colares, A.- Stability of hypersurfaces with constant r-mean curvature, Annals of Global Analysis and Geometry 15, 277-297 (1997). MR 98h:53091

3. Barbosa, J. L. and do Carmo, M. - Stable minimal surfaces. Bull. Amer. Math. Soc. 80, 581-583 (1974). MR 49:1307.

4. Bernstein, S. - Sur un théorème de Géométrie et ses applications aux équations aux dérivées partialles du type elliptique. Comm. de la Soc. Math. de Kharkov (2ième sér.) 15, 38-45 (1915/1917).

5. Bullen, P.S., Mitrinovic, D.S. and Vasic, P.M. - Handbook of Means and Their Inequalities, Translated and revised from the Serbo-Croatian. Mathematics and its Applications (East European Series) 31. D. Reidel Publishing Co., Dordrecht, 1988. MR 89d:26003

6. De Giorgi, Ennio - Una estensione del teorema di Bernstein. (Italian) Ann. Scuola Norm. Sup. Pisa (3) 19, 79-85 (1965). MR 31:2643 
7. Hardy, G., Litlewood, J. and Polya, G. - Inequalities, $2^{\text {nd }}$ Ed. - Cambridge Univ. Press, 1989. MR 89d:26016

8. Hounie, J. and Leite, M.L. - Two-ended hypersurfaces with zero scalar curvature. Indiana Univ. Math. J. 48, 867-882 (1999). MR 2001b:53077

9. Nomizu, K. and Smyth, B. - On the Gauss mapping for hypersurfaces of constant mean curvature in the sphere. Comment. Math. Helv. 44, 484-490 (1969). MR 41:2588

10. Reilly, R.C. - Extrinsic rigidity theorems for compact submanifolds of the sphere, J. Diff. Geom. 4, 487-497 (1970). MR 44:7480

11. Reilly, R.C. - Variational properties of functions of the mean curvatures for hypersurfaces in space forms, J. Diff. Geom. 8, 465-477 (1973). MR 49:6102

12. Rosenberg, H. - Hypersurfaces of constant curvature in space forms. Bull. Sci. Math. 117, 211-239 (1993). MR 94b:53097

13. Simons, J. - Minimal varieties in riemannian manifolds. Ann. of Math. (2) 88, 62-105 (1968). MR 38:1617

Departamento de Matemática, Universidade Federal de Alagoas, 57072-900, Maceió, AL, BRAZIL

E-mail address: hilario@mat.ufal.br

Institut de Mathématiques de Jussieu, 2 Place Jussieu, 75251 Paris, France

E-mail address: rosen@math.jussieu.fr

Departamento de Matemática, Universidade Federal do Rio de Janeiro, Caixa Postal 68530, 21945-970 Rio de Janeiro, RJ, Brazil

E-mail address: walcy@im.ufrj.br 